\title{
EL APRENDIZAJE TOTAL DE LOS CONCEPTOS CIENTÍFICOS ÁCIDO-BASE
}

\author{
Sara Zafra A. \\ Instituto Ciudad Jardín del Norte
}

\begin{abstract}
This paper pretends to communicate the results after to applicate a pedagogical and didactical strategy to global learning process in scientific concepts such as acid - base, with Institueo Ciudad Jardin del Norte's students of $10^{\text {th }}$ level located in $11 t^{\text {h }}$ locality of Suba of Bogotá city. The strategy is based in different activities which pretended that the implicated subjects into the teaching - learning process got a triple recognition: that they recognized meanings own created by themselves, how was their creation process and the most important, that they identified themselves into those meanings; so they would have the possibility with this triple recognition to identify with the cause of cognitive activity and answered by the consequences of their own learning.
\end{abstract}

\section{RESUMEN}

El presente artículo pretende comunicar los resultados obtenidos en el trabajo de Investigación El Aprendizaje Total de los conceptos científicos ácido-base”, realizado con estudiantes de grado décimo del Instituto Ciudad Jardín del Norte, ubicado en la localidad 11-Suba de la ciudad de Bogotá. La estrategia pedagógica y didáctica centrada en una serie de actividades, pretendió que los sujetos involucrados en el proceso enseñanzaaprendizaje reconocieran los significados que iban elaborando, reconocieran cómo los construían y por supuesto se reconocieran en dichos significados; de tal manera que este triple reconocimiento les posibilitara identificarse con aquello que produce la actividad cognoscitiva y respondieran por las consecuencias de su propio aprendizaje.

\section{INTRODUCCIÓN}

La investigación se constituye en un aporte al campo pedagógico y didáctico en la enseñanza de las ciencias, en la medina que surge como una preocupación de revisar, reflexionar y mejorar la practica pedagogía que seguramente redundará en mejorar los procesos enseñanza-aprendizaje a través del concepto de aprendizaje total: una aproximación teórica (Gallego B., Pérez M., Torres de G. L. N.,1997) y posibilitará contrastar los principios del programa de investigación Representaciones y Conceptos científicos" (Gallego B. R. y Perez M., R., 1994).

Para esto, se pensó en el aprendizaje total de los conceptos científicos acidobase, estos conceptos al igual que otros, posibilitan explicar fenómenos de la vida diaria. El aprendizaje total involucra una transformación conceptual, metodológica, actitudinal y 
axiológica del sujeto quien paulatinamente se va involucrando y comprometiendo de acuerdo a su propio proyecto ético de vida.

Se inició con una revisión bibliográfica, para saber hasta dónde se había trabajado en relación con las transformaciones conceptuales, metodológicas, actitudinales y axiológicas de los estudiantes. En consecuencia, se encontró que algunas investigaciones demuestran que el proceso enseñanza-aprendizaje de los conceptos acidobase, se ha abordado desde lo conceptual, lo metodológico y lo actitudinal, pero como unidades separas entre sí, prevaleciendo mas las influencias de tipo conceptual y metodológico que las de tipo actitudinal y axiológico.

En cuanto al aspecto conceptual, De la Guardia. CM., Salvador,C.A, López Calafi, y otros (1985); Alarcón, A., Espitia,J.G., y Muñoz R., (1988) y Lardanca, M. y otros (1993), consideraron que las concepciones alternativas que manejan los estudiantes sobre los conceptos ácido-base, son difíciles de erradicar después de la instrucción y que el concepto ácido-base lo explican con mayor facilidad que el de base.

En el aspecto actitudinal. González García,. F M (1992) en su investigación concluye que la aplicación de metodologías activa favorece una actitud positiva hacia las ciencias y por supuesto. hacia el aprendizaje de los conceptos científicos. Con estas metodologías los estudiantes explican con mayor facilidad los fenómenos ácido-base utilizando los conceptos de Bronsted-Lowry que el concepto de Lewis.

En relación con el aspecto metodológico Nakhleh M. Y Krajcik J. (1993) afirmaron que, el laboratorio provee un ambiente de aprendizaje que conduce a integrar la teoría con los fenómenos físicos observados durante la manipulación del equipo de laboratorio. y Ericson y Simón (1984) consideraron que el acceso a mas información dada por los instrumentos tecnológicos del laboratorio, los estudiantes son capaces de explicar los conceptos ácido-base en forma mas analítica

Como estrategias para la reconstrucción y construcción de significados, formas de significar y de actuar en relación con los conceptos ácido-base, Pérez Miranda R. (1991) utilizó la UVE heurística y el mapa conceptual concluyendo que estos instrumentas combaran en el proceso, poro que aun falta puntualizar mas para llegar a conclusiones más validas. Otros investigadores París, M , (1989) y González García, J. J., (1992), concluyeron que los mapas conceptuales permiten desarrollar en los estudiantes capacidades intelectuales como las de análisis y de síntesis.

Esto nos permitió pensar que aún hace falta investigar sobre las transformaciones conceptuales, metodológicas, actitudinales y axiológicas como una unidad imbricada. Es decir, al modificarse una necesariamente se modifican las otras. Por consiguiente, se pensó que el enfoque del aprendizaje total podría dar resultados mas satisfactorios en términos de la eficiencia y de la eficacia de una enseñanza y de un aprendizaje más científicos, y retomar los mapas conceptuales como instrumentos que dan cuenta de dichas transformaciones.

El presente articulo pone a consideración de la comunidad académica, los resultados obtenidos en la aplicación de la estrategia pedagógica y didáctica centrada en una serie de actividades para el aprendizaje total de los conceptos científicos ácido-base. 


\section{OBJETIVO}

El objetivo principal del estudio consistió en diseñar y aplicar una estrategia pedagógica y didáctica, fundamentada en los principios del programa de investigación Representaciones y Conceptos Científicos", de modo que posibilitara el aprendizaje total de los conceptos ácido-base en los estudiantes.

\section{PLANTEAMIENTO DEL PROBLEMA}

Se evidencia que la reconstrucción y construcción de nuevas formas de significar y de actuar en relación con los conceptos científicos, en nuestro caso, los conceptos ácidobase, es un proceso complejo, que requiere de un modelo integrador tanto de los aspectos conceptual, metodológico, actitudinal como del axiológico.

Además, es importante considerar que el estudiante en su proceso de aprendizaje, se involucra y compromete como persona de acuerdo con las relaciones que establece con los otros, por los otros, para coexistir con los demás y para pertenecer a ese colectivo. Y por supuesto, para luego dar cuenta de eso que sabe, de cómo lo sabe, por qué lo sabe y qué beneficios y dificultades le ha traído su saber (Gallego B.R.,y Pérez M.R.,1996).

En consecuencia, se hace necesario el ordenamiento del ámbito pedagógico y didáctico que permita la integración y el aprendizaje total de los conceptos ácido-base.

Parlo tanto, se abordé la siguiente problemática: ¿En qué medida la estrategia pedagógica y didáctica centrada en una serie de actividades permite que los estudiantes se acerquen al aprendizaje total de los conceptos científicos ácido-base, teniendo en cuenta lo que ellos ya saben y la posibilidad de negociar significados para llegar a los respectivos acuerdos conceptuales, metodológicos, actitudinales y axiológicos?

\section{HIPÓTESIS}

Si la estrategia diseñada como ordenamiento del ámbito pedagógico y didáctico permite negociar significados, formas de significar y de actuar en los estudiantes, entonces, se logrará el aprendizaje total de los conceptos científicos ácido-base y por supuesto, habrá también un cambio en las estructuras conceptuales, metodológicas, actitudinales y axiológicas (ECMAAS) de cada uno de ellos.

\section{MARCO CONCEPTUAL}

Este trabajo se apoyó en los principios del Programa de Investigación Representaciones y Conceptos Científicos" Gallego BR. y Pérez M.R. (1994) y en el concepto de aprendizaje total: una aproximación teórica, Gallego BR., Pérez M.R. y Torres de Gallego L.N. (1997).

El Programa de Investigación está basado en la propuesta de Lakatos (1983) con la Metodología de los Programas de Investigación Científica. Lakatos considera que todo programa tiene un núcleo firme, que está constituido por los postulados científicos centrales, desde los cuales se deriva el cinturón protector que lo constituye el conjunto de hipótesis que se pretenden contrastar. Dicho programa de investigación está dotado de reglas metodológicas las cuales nos dicen las rutas que deben seguirse (heurística positiva) y las que deben evitarse (heurística negativa). Este poder heurístico permite evaluar los programas de investigación que continuamente están en competencia. Por lo 
tanto, la heurística negativa se entiende como la tendencia a conservar el programa, es decir, a no modificar el núcleo firme y la heurística positiva como un conjunto de hipótesis auxiliares compatibles con el núcleo firme las cuales son susceptibles de cambio y de modificaciones.

De esta manera los principios del Programa de Investigación Representaciones y Conceptos Científicos" constituyen el núcleo firme que forman el cuerpo conceptual, metodológico, actitudinal y axiológico, los cuales se presentan a continuación:

- Primero: Todos los seres humanos en comunidad construyen representaciones de sí mismos, de la naturaleza y de la sociedad. Estas representaciones son organizadas en unas Estructuras Conceptuales, Metodológicas, Actitudinales y Axiológicas (ECMAAs), desde las cuales ordenan su interioridad como personas y la exterioridad con miras a una actuación para la intervención, el control y el dominio de la misma actuación.

- Segundo: Los seres humanos, sin excepción alguna, nacen en un mundo social, económico y político ya ordenado. Ese orden es el que les posibilita su autoconstrucción como personas y el que les da sentido de pertenencia.

- Tercero: Entre lo que cada individuo construye y el medio sociocultural, siempre hay una relación de intercambio, de interacción mutua, es decir, de negociación.

El concepto de Aprendizaje Total como aproximación teórica hace referencia a que cada estudiante se involucre y se comprometa en el proceso de cambio conceptual, metodológico, actitudinal y axiológico a través del triple reconocimiento: reconozca el saber que sabe, las maneras como ha llegado a ese saber y se reconozca que ha sido él quien lo ha construido, reconociéndose a su vez en lo que sabe.

De esta manera con el proceso de aprendizaje total se van reconstruyendo y construyendo nuevos significados, formas de significar y de actuar y, en la medida en que esto se va dando, la forma de aprender se va transformando al mismo tiempo y, por tanto, las maneras de involucrarse y de comprometerse.

\section{MARCO METODOLÓGICO}

Esta es una investigación que se plantea desde la realidad escolar, con la participación del docente y de los estudiantes. Es una investigación de tipo cualitativo centrada en acuerdos sociales, donde las creencias determinan lo que debe contarse como hechos; por lo tanto, involucra un proceso activo, sistemático y riguroso, en donde el investigador (docente) entra en el campo con una orientación teórica consciente (Principios del Programa Representaciones y Conceptos Científicos" y el concepto del "Aprendizaje Total") y desde la cual sugiere las preguntas que orientarán la investigación (problema de investigación).

En el desarrollo se tuvieron en cuenta las representaciones que habían construido los estudiantes en relación con los conceptos científicos ácido-base y desde ellas determinar las transformaciones conceptuales, metodológicas, actitudinales y axiológicas de los estudiantes en relación con el aprendizaje total de los conceptos científicos ácido-base. Para analizar la información se utilizaron los cuestionarios, la prueba tipo Likert y los mapas conceptuales. 
Los cuestionarios con preguntas abiertas permitieron a los estudiantes dar cuenta de su propio saber, los cuales se aplicaron al comienzo y al final del proceso. La información recolectada se sistematizó de acuerdo a unas categorías construidas, las cuales se enuncian a continuación.

Las representaciones eran concepciones alternativas ( Furió M. C., 1996) desde lo fenomenológico (CAF), cuando se referían a situaciones del contexto cotidiano y a expresiones de sentido común. Así mismo, las representaciones eran concepciones alternativas desde lo químico (CAQ), cuando se expresaron utilizando lenguaje científico sin coherencia significativa y eran concepciones científicas (CC) cuando se acercaban a las que maneja la comunidad de los químicos.

Para identificar las representaciones de los conceptos científicos ácido-base construidas por los estudiantes, se formuló la pregunta: ¿Qué saben de los conceptos ácidobase?, la cual se aplicó en la fase inicial, intermedio y final del proceso.

Las representaciones actitudinales y axiológicas de los estudiantes en relación con las conceptualizaciones de ácido-base en la conservación del ambiente, se evaluaron a través de las preguntas: ¿Los conceptos ácido-base posibilitan comprender situaciones relacionadas con los componentes del planeta tierra?. Explique. ¿Aprender los conceptos científicos como los de ácido-base colaboraría en acciones positivas frente al ambiente y a su cuerpo? ¿Por qué?

La prueba tipo Likert (Gene E. S., 1978), empleada en la sistematización de los resultados, es una escala en la que el valor que obtiene cada estudiante resulta de la suma de las respuestas a diversas proposiciones. En esto caso, la prueba consistió en 36 proposiciones referidas a los cuatro componentes: conceptual, metodológico, actitudinal y axiológico que involucran el aprendizaje total de los conceptos científicos ácioo-base y que se pudieron clasificar como favorables o desfavorables. (Ver anexo).

Las proposiciones utilizadas como ítems de la escala se presentaron con cinco opciones de respuestas y asignándoles arbitrariamente un número así: 1 . Totalmente de acuerdo 2. De acuerdo. 3. No se qué decir. 4. En desacuerdo, y 5. Totalmente en desacuerdo. Estos valores se invirtieron para items redactados negativamente (Saavedra M.J,1996).

Los mapas conceptuales son una estrategia propuesta por Novak y Gowin, 1988 quienes se apoyaron en la teoría del aprendizaje significativo de Ausubel, 1995. Las relaciones jerárquicas permitieron determinar qué conceptos eran más relevantes, cuáles subordinados y qué relaciones establecieron entre ellos. Dieron cuenta del pensamiento activo, de la reorganización cognitiva y del grado de diferenciación de los conceptos construidos.

Para elaborarlos se procedió de la siguiente manera: se escogieron los conceptos a partir del tema general acidez-basicidad o dando la lista previamente, luego se procedió a jerarquizarías, empleando conectares a través de los cuales explicitaron el sentido de la relación, es decir, formaron una proposición con sentido y finalmente lo socializaron para contrastar, negociar y construir los acuerdos finales.

La investigación se realizó en el año 1997 en el Instituto Ciudad Jardín del Norte con 68 estudiantes entre 14 y 16 años de edad de los géneros masculino y femenino del grado décimo, agrupados en dos cursos 10.1 y 10.2 . 
A continuación se presenta la estrategia pedagógica y didáctica que consistió en una serie de actividades:

- La primera tuvo que ver con La motivación. Gagné (1979), afirma que el aprendizaje se produce cuando el individuo está motivado.

Mediante una lectura individual sobre el concepto de Aprendizaje Total, los estudiantes respondieron a los siguientes interrogantes: ¿qué es aprender?, ¿cómo se aprende?, ¿para qué se aprende?

- Una vez que los estudiantes estaban motivados, se les preguntó qué sabían del tema. Con la participación de todos se escribió en el tablero la lista de conceptos: óxido, sal, base, agua, $\mathrm{pH}$, ácido, $\mathrm{HCL}$, ión. $\mathrm{NaOH}$, solución, equilibrio, Arrhenius. Luego, los estudiantes relacionaron estos conceptos jerárquicamente en un mapa conceptual, trabajo que se hizo primero individualmente. Después, en grupos, lo cual les permitió contrastar las formas de significar y de actuar que hasta ahora tenían al respecto. En un segundo momento, volvieron a elaborar otro mapa conceptual en el cual involucraron otros conceptos como sustancia, ionización, reacción, neutralización, Lewis, Bronsted-Lowry, amoniaco. Este también lo presentaron en plenaria a todo el curso para la negociación de significados y finalmente llegar a acuerdos conceptuales, metodológicos, actitudinales y axiológicos.

- En relación con la actitud se aplicó la prueba tipo Likert la cual permitió detectar la situación inicial y final de los estudiantes frente a sus ECMAAs.

- De igual manera, cobró un especial significado la bibliografía que los estudiantes consultaron sobre la temática, así como también fueron relevantes los espacios de socialización los cuales permitieron confrontar lo que sabían, las maneras de saberlo y finalmente llegar a los respectivos acuerdos conceptuales, metodológicos, actitudinales y axiológicos.

- Entre otras herramientas metodológicas que se emplearon se encuentran los mapas conceptuales a través de los cuales los estudiantes jerarquizaron y relacionaron los conceptos, de esta manera pudieron reconstruir y construir nuevas formas de significar y de actuar. Así mismo, la experimentación se constituyó en una posibilidad de constrastar las hipótesis formuladas, fortaleciendo la reconstrucción y construcción de los significados en relación con los conceptos científicos ácido-base.

- Las preguntas de los cuestionarios al comienzo y al final del proceso colaboraron para que los estudiantes relacionaran y aplicaran los conceptos con el mundo de la vida.

\section{RESULTADOS}

La investigación permite concluir entre varios aspectos los siguientes:

En relación con la motivación, reconocer el qué, el cómo, el por qué y el para qué se aprende, a los estudiantes les posibilitó reflexionar, ser conscientes de su propio aprendizaje y por supuesto, tener una actitud positiva hacia la nueva experiencia de aprendizaje. Para ellos aprender es un compromiso personal que desde la interpretación de la información se llega a conocer y a saber más. Esto se evidenció en las respuestas dadas a las preguntas de motivación. 
Como ejemplo se presentan las ideas del estudiante $\mathrm{N}^{0} 6$ quien respondió: "aprender es una actividad individual que me compromete como persona a crear mi propia vida, pero también se aprende de los demás. Se aprende porque la persona quiere, mirando nuestros errores, poniendo empeño, dando y recibiendo y se aprende para tener conocimiento para luego tener respuestas a nuestras inquietudes y aplicarlas en nuestra vida práctica".

Reflexionar sobre el aprendizaje desde estas preguntas colaboré de alguna manera a que cada estudiante reconociera los significados que había construido, las maneras como había llegado a saber y reconociera que fue él quien construyó dichos significados.

Se evidencié aprendizaje total respecto a los conceptos científicos ácido-base en la mayoría de los estudiantes que participaran en la investigación, sin embargo el cambio en las estructuras conceptuales, metodológicas, actitudinales y axiológicas no fue de manera directa, lineal ni en el mismo nivel para cada uno de los estudiantes.

Quiere decir que el aprendizaje total implica una transformación en las estructuras pera de manera diferencial. Es decir, cada quien significa desde sus ECMAAs, y las maneras de significar son diferentes y están influenciadas por el contexto social, económico, familiar y por las intenciones de su propio proyecto ético de vida.

En relación con las transformaciones de las estructuras conceptuales, metodológicas, actitudinales y axiológicas se pudo establecer lo siguiente:

Las representaciones conceptuales que los estudiantes habían construido cuando se inició el aprendizaje total de los conceptos científicos ácido-base fueron más de tipo concepción alternativa desde la fenomenológico y químico (CAF;CAQ) que concepciones científicas (CC). La fenomenológico hacía referencia a los beneficios y perjuicios que tanto los ácidos como las bases ocasionan al hombre; desde lo químico, definieron a los ácidos y a las bases como una clase de óxidos, como algo que tiene que ver con los iones en solución, como producto de una reacción, como una unión, como una combinación, es decir, son representaciones que aún no íntegra en las estructuras mentales el lenguaje científico para establecer relaciones válidas.

En el proceso inicial para la mayoría de los estudiantes, se pudo establecer que sus ECMAAs carecen de un modelo conceptual coherente que les permitiera construir un significado válido de los conceptos científicos ácido-base, aunque se evidencié una incipiente intencionalidad de relacionar estas conceptos entre sí y con el significado de otros conceptos de la química inorgánica.

Se presentan los textos escritos del estudiante $\mathrm{N}^{\circ} 6$.

Para este estudiante, inicialmente ácido es una clase de óxido, es la oxidación producida por una combinación y base es una división de los óxidos'.

Después de la consulta bibliográfica y del recuento histórico, en la fase intermedia, el mismo estudiante responde: Los ácidos al reaccionar con las bases producen una sal más agua, los ácidos son de sabor amargo y se encuentran en los alimentos como el vinagre, la maracuyá y el limón, resultan de la combinación de un no metal con el oxigeno, se clasifican en hidroxiácídos y según Arrhenius en solución acuosa producen iones $\mathrm{H}^{+}$y las bases algunas son resbalosas y venenosas, tienen sabor a lejía”. 
Y al final del proceso, después de participar activamente en las discusiones, de la experimentación y de la elaboración de mapas conceptuales el estudiante responde ante la misma pregunta lo siguiente: La acidez de las soluciones se determina utilizando unos indicadores, los más conocidos son la fenoftaleina, naranja de metilo, tornasol, indicador universal y el azul de bromotimol. Una solución es ácida cuando su grado de $\mathrm{pH}$ es menor que 7. Según Arrhenius un ácido en solución acuosa produce iones $\mathrm{H}^{+}$Las soluciones ácidas conducen la corriente eléctrica por medio de la ionización que es un proceso de ceder y captar iones que son átomos con carga eléctrica. Y una sustancia básica produce en solución acuosa iones $\mathrm{OH}$ y al medir su pH es mayor que 7. La basicidad de una solución se determina con unos indicadores al igual que para los ácidos. La tfnoftaleína cambia a color púrpura y el papel tornasol a azul cuando hacen contacto con soluciones básicas".

En él se evidencia que las representaciones conceptuales que sobre ácido-base había construido como concepciones alternativas desde lo fenomenológico o desde lo químico, cambiaron a unas representaciones más cercanas a las que maneja la comunidad de especialistas. Esto significa que las transformaciones en las ECMAAs es un proceso paulatino, que evoluciona de acuerdo a los intereses, necesidades e intencionalidades de cada quien y que se da en relación consigo mismo, con los demás y con el entorno natural, social, cultural y político.

Los resultados de la prueba tipa Likert mostraron que las ECMAAs de la mayoría de los estudiantes (61) de una posición de indiferencia en los aspectos conceptual, metodológico y actitudinal y una posición tendiente a estar de acuerdo en el axiológico, se transformó a una posición cercana a estar de acuerdo en los aspectos conceptual, actitudinal, el aspecto axiológico sigue igual y el metodológico cambió a una posición de indiferencia.

En cuanto a lo metodológico, la transformación en la mayoría de Is estudiantes no fue significativa, esto pudo deberse a que las estructuras de significado (ECMAAs) se encontraban en un estado de desequilibrio, ya que la actividad cognoscitiva sigue trayectorias no definidas y caóticas, en la que la elección del punto de bifurcación (alternativa de solución) está mediada por los intereses de cada quien. Además, debido a la complejidad del proceso, no se esperaba que los componentes de las ECMAAs se transformaran a la vez y en el mismo sentido; sino que si uno de ellos se transforma, implicara necesariamente, la transformación paulatina de los otros y el posicionamiento del triple reconocimiento.

Para el estudiante $\mathrm{N}^{0} 6$ el cambio más significativo se evidencié en el aspecto actitudinal de una posición de indiferencia pasó a estar de acuerdo. Aspecto actitudinal que también se observó cuando desde el autoreconocimiento de sus insuficiencias explicativas en relación con los conceptos científicos ácido-base, decide no asistir a clase para ir a la biblioteca a consultar sobre óxidos porque considera que si entiende bien este concepto puede comprender mejor los de ácido-base. Esto contrasta que las representaciones de un concepto se apoyan en otras representaciones.

La puntuación de los mapas conceptuales permitió interpretar que hubo un aumento gradual en el número de jerarquizaciones, en el número de conceptos y en el número de relaciones válidas entre los mismos. Además, que en las ECMAAs de los estudiantes los conceptos científicos ácido-base sufrieron una diferenciación progresiva, la cual se reconoció cuando en los mapas se establecen vínculos preposicionales con otros 
conceptos. Sin embargo la reconciliación integradora se evidencié sólo en algunos estudiantes que elaboraron relaciones cruzadas entre algunos conceptos.

También los diferentes mapas muestran qué conceptos jerarquizaban inicial-mente y cuáles al final. Así, inicialmente los conceptos agua y óxido estaban en primer nivel de jerarquización y al final del proceso lo ocuparon los conceptos científicos ácido-base. Esto demostró que los conceptos científicos ácido-base en las ECMAAs empezaron a ser inclusivos y más amplios, es decir, para la mayoría de los estudiantes dichos conceptos adquirieron más significado.

La elaboración de mapas conceptuales fue una actividad que ayudé a fomentar la creatividad (diversidad de formas de presentación). La socialización de los mismos posibilité la negociación de significados, formas de significar y de actuar que llevó a los respectivos acuerdos conceptuales, metodológicos, actitudinales y axiológicos. En dicha socialización cobré sentido respetar las ideas de los demás y reconocer las debilidades y las fortalezas de sus interpretaciones. Fue un proceso en el que la mayoría de los estudiantes que participaron pudieron darse cuenta de qué sabían, cómo sabían y cómo fueron cambiando las maneras de saber y por supuesto las de actuar en consecuencia con lo que ya sabían. De igual manera, pudieron reconocer-se en sus propias elaboraciones cuando pudieran aceptar que su mapa tenía más o menos relaciones válidas.

Para el estudiante $\mathrm{N}^{0} 6$, el primer mapa conceptual presenta solo una proposición "Los óxidos se dividen en base y ácidos", que refleja una concepción alternativa desde lo químico. De igual manera en el segundo mapa sigue utilizando concepciones alternativas desde lo químico como "Los ácidos son una reacción de un elemento metálico con la disociación de sustancias" Pero ya hace una reconciliación integradora entre dos conceptos, Las bases neutralizan a los ácidos". En el tercer mapa se evidencia que la representación de los conceptos científicos ácido-base se apoya en otras representaciones como las de óxido y el carácter metálico y diferencia das conceptos el de Arrhenius y el de Lewis. "Los óxidos metálicos al combinarse con el agua producen las bases que son sustancias que aceptan protones según Lewis, neutralizan los ácidos, vuelven azul el papel tornazol y roja la fenoftaleina".

En este estudiante se evidencia que a medida que se avanza en el proceso enseñanza-aprendizaje las formas de significar y de actuar van cambiando, parlo tanto, las ECMAAs también. Por eso en el tercer mapa aparece un aumento gradual en el número de jerarquizaciones, en el número de conceptos y en el número de relaciones válidas entre los mismos.

Finalmente, los resultadas de la información de los instrumentos utilizados en la investigación (cuestionarios, prueba tipo Likert y mapas conceptuales) nos permitieron contrastar que el aprendizaje total de los conceptos científicos ácido-base es una actividad idiosincrática que se logra desde el ordenamiento del ámbito escolar, para que cada quien transforme sus ECMAAs.

Esta transformación posibilitó que cada estudiante reconociera las relaciones mutuas entre los conceptos ácido-base y de éstos con otros de la química. Reconocieran que el diálogo, la discusión y la controversia, como procesos de negociación en el trabajo grupal, permiten establecer acuerdos conceptuales, metodológicos, actitudinales y axiológicos, en torno a lo que cada quien significa, la forma como significa y actúa. Además, que 
reconocieran la importancia que dentro del proceso tiene el respeto parlas formas de significar y de actuar de los otros en un ambiente de tolerancia.

Aunque no todos los estudiantes lograron cambiar positivamente sus ECMAAs en tanto que el ordenamiento del ámbito pedagógico y didáctico no favorece de igual manera a todos los estudiantes, se pudo concluir que la mayoría de los estudiantes construyeron una representación de los conceptos científicos ácido-base teniendo en cuenta la interacción que se dio entre ácido y base para producir una neutralización, la interacción entre sustancia-energía, explicando La ionización del ácido o de la base en agua y la relación que establecieron con el componente matemático, cuando expresaron la ionización de dichos conceptos mediante la ecuación química correspondiente aunque no se llegó a la constante de equilibrio. El componente tecnológico de los conceptos científicos ácido-base se hizo evidente cuando en los experimentos utilizaron indicadores, pHmetros, material de vidrio y otros para determinar con exactitud el valor de la acidez 0 basicidad de los materiales escogidos para la respectiva clasificación.

\section{CONCLUSIONES}

La estrategia específica diseñada como ordenamiento del ámbito pedagógico y didáctico, posibilité que los estudiantes negociaran significados, formas de significar y de actuar para llegar a acuerdos conceptuales, metodológicos, actitudinales y axiológicos en relación con los conceptos ácido-base.

El concepto de aprendizaje total cobra sentido en ja medida en que permite sustentar y orientar una estrategia pedagógica y didáctica para el aprendizaje de los con-coptos científicos, tal como se evidencia en las transformaciones observadas en los estudiantes que participaron en la investigación. Transformaciones que avanzaron desde jas concepciones alternativas fenomenológicas hasta aproximarse a las que maneja la comunidad de especialistas. Podría pensarse que con la interdisciplinariedad entre las áreas y en mayor tiempo, la propuesta pedagógica aportaría valiosos resultados.

Las representaciones iniciales que los estudiantes tenían sobre los conceptos científicos ácido-base se transformaron gracias a que la estrategia diseñada posibilité en cada uno de ellos, de acuerdo con sus intereses, el triple reconocimiento: reconocer el saber que había construido, los procesos mediante las cuales llegó al nueva saber y las nuevas significados, formas de significar y de actuar en relación con dichos conceptos.

En cuanto al análisis e interpretación de los resultados obtenidos al aplicar los diferentes instrumentos para evaluar las transformaciones en las ECMAAs de los estudiantes, se puede concluir que:

- La prueba tipa Likert permitió evidenciar que los aspectos conceptual, motadológico, actitudinal y axiológico referidos a las conceptos científicas ácido-base como unidad imbricada no se transformaron a la vez. El ordenamiento del ámbito pedagógica y didáctico no permitió una transformación significativa del aspecto metodológico; aunque a lo largo del proceso de evolución del sujeto se fomentará. Podría pensarse en aproximar la propuesta pedagógica a transformar este aspecto.

- Los mapas conceptuales brindaron información sobre el aumento gradual en el número de jerarquizaciones, en el número de conceptos y en el número de relaciones válidas entre los mismos, que la mayoría de los estudiantes construyeron, más no un 
aumento en las relaciones cruzadas y en el número de ejemplos. Esto significa, que la estrategia permitió que en Las ECMAAs de los estudiantes se diera una diferenciación progresiva entre los conceptos pero, habría que analizar, de qué manera podría esta estrategia colaborar en el desarrollo de la reconciliación integradora, para próximas investigaciones.

- Los escritos correspondientes a las respuestas de las preguntas abiertas de los cuestionarios, permitieron evidenciar un avance significativo en el aprendizaje total de los conceptos científicos ácido-base, desde las definiciones iniciales hasta una verdadera conceptualización, cercana a la que maneja la comunidad de los químicos. Estos escritos que reflejan las transformaciones en el lenguaje y que argumentan el saber construido por cada sujeto durante el proceso pedagógico y didáctico.

Dentro del aprendizaje total, se considera que cada estudiante significa desde sus ECMAAs y desde las intenciones de su propio proyecto ético de vida, por lo tanto, las aproximaciones al aprendizaje total de los conceptos científicos ácido-base que se evidenciaran en el grupo de los 68 estudiantes que participaron en ja investigación no todas fueran iguales. Esto contrasta que el aprendizaje total de los conceptos científicas es una actividad idiosincrática y que las construcciones conceptuales, metodológicas, actitudinales y axiológicas cada vez van adquiriendo cierto grado de validez.

El ordenamiento del ámbito es esencial para favorecer un aprendizaje que contemple lo conceptual, metodológico, actitudinal y axiológico. Inicialmente la actividad de motivación y la prueba tipo likert fueron los motores que impulsaron el aprendizaje total de los conceptos científicos ácido-base

Frente a la hipótesis, puede decirse que se canstrastó positivamente, ya que se permitió que cada uno de los estudiantes negociara significados y se establecieran entre el grupo acuerdas conceptuales, motado-lógicos, actitudianles y axiológicos, siendo conscientes de la importancia que tiene el involucrarse y comprometerse en su propio aprendizaje.

En términos generales, podría afirmarse que se logré una aproximación al aprendizaje total de los conceptos científicos ácido-base, al propiciarse el triple reconocimiento en los estudiantes, lograr una transformación a nivel conceptual, actitudinal y axiológica y al extrapolar el saber construido con situaciones de la vida diaria. Es importante anotar aquí, que las construcciones elaboradas por los estudiantes no involucran toda la red conceptual que la comunidad de especialistas maneja, parlo que el concepto de Arrhenius fue el que mas significado tuvo para los estudiantes.

\section{BIBLIOGRAFIA}

Alarcón A., Espitia J.,y Muñoz G., 1988."Estudio de las incidencias de las ideas intuitivas en la aprehensión de los conceptos científicos de ácido y base". En: Alumnos de Grado Décimo de Educación Media. Universidad Pedagógica Nacional. Facultad de Ciencia y Tecnología. Departamento de Química. Santa Fe de Bogotá.

Ausubel D., Novak J., Hanesian H., 1995. "Psicología educativa. Un punto de vista Cognoscitivo”. México. Octava reimpresión. Editorial Trillas. 
De la Guardia C. M., Salvador C.A., López C., y otros, 1985. "Errores conceptuales en la comprensión de los equilibrios ácido-base". Enseñanza de las Ciencias, Número extra, pág. 61.

Furió Mas Carlos J., 1996. "Las concepciones alternativas del alumnado en ciencias: Dos décadas de investigación". Resultados y Tendencias". Alambique. N9. Año III, págs. 711

Gagné, FR. (1979). Las condiciones del aprendizaje”. Tercera edición. Interamericana SA. México.

Gallego Badillo, R. y Pérez Miranda, R., 1994. "Representaciones y conceptos científicos" Un programa de investigación de la Universidad Pedagógica Nacional, Facultad de Ciencia y Tecnología, Departamento de Química. U.P.N. Bogotá.

Gallego Badillo, R.; Pérez Miranda, R. Y Torres de Gallego, L. N., 1997. "El concepto de aprendizaje total: Una aproximación teórica”. Revista Estudios en Pedagogía y Didáctica. Departamento de Química. U.P.N. Bogotá.

Gene E. Summers, 1978. Medición de actitudes, México: Editorial Trillas.

González García, F. M., 1992, "Los mapas conceptuales de J.D. Novak como instrumentos para la investigación en Didáctica de las Ciencias Experimentales". Enseñanza de las Ciencias 10(2), 148-158.

Lakatos, Y. 1983. La metodología de los Programas de Investigación Científica. Madrid. Alianza Editorial.

Lardanca, M.; Nieto, M. y Rodriguez, M., 1993. "Evolución de los conceptos ácido-base a lo larga de la enseñanza media”. Enseñanza de las Ciencias. 11(2), 125-129.

Nakhleh, Mary., y Krajcik Joseph., 1993. "A Protocol Analysis of the influence of Technology on Students' Actions, Verbal Commentary, and Thought Processes during the Performance of Acid-Base Titrations". Journal of Research in Science teaching. Vol. 30 , No. 9 pp. 1.149-1.168.

Novak, J.D. 1988. “ Constructivismo humano: Un consenso emergente”. Enseñanza de las Ciencias, 6 (3), 213-223.

Novak, J., y Gowin, D., 1984. 'Aprendiendo a aprender”. Barcelona, España: Ed. Martínez Roca.

París, M. 1989. ' Esquemas conceptuales en torno a los ácidos y las bases. Implicaciones de la instrucción en el proceso evolutivo de estos conceptos". Enseñanza de las Ciencias, No. Extra, III Congreso, Tomo 2. pp. 290-293.

Pérez Miranda, R., 1991. "Aprendizaje de los conceptos de ácido y de base en estudiantes de educación media vocacional: Una estrategia pedagógica fundamentada en la Teoría de Novak sobre la Educación". Trabajo de Grado. Universidad Pedagógica Nacional. Facultad de Ciencia y Tecnología. Departamento de Química. Docencia de la Química. Santafé de Bogotá. 
Pérez M., R.; Gallego, B. R.; Torres de Gallego, L. N., 1996. "El problema de la formación científica" Revista Investigación Educativa, Agosto, No 11. Pp. 22-25.

Pérez M. R.; Gallego, B. R., 1995. Corrientes constructivistas. Bogatá, Colombia: Editorial Magisterio, Colección Mesa Redonda.

Saavedra Martha J., 1996. Métodos para la medición de actitudes. Boletín PPDQ, No. 16, pp. 15-19.

\section{ANEXO \\ PRUEBA TIPO LIKERT”}

A continuación aparecen una serie de afirmaciones relacionadas can los conceptos científicos Ácido-Base, las cuales están numeradas de 1 a 36 y sobre las que se pide su posición de conformidad con la siguiente escala de valoración:

1. Totalmente de acuerdo. 2. De acuerdo. 3. No se que decir. 4. En desacuerdo. 5. Totalmente en desacuerdo.

1. Un ácido es una sustancia química que acepta un par de electrones.

2. El aprendizaje del concepto ácido base me permite desarrollar habilidades cognoscitivas y manuales.

3. Los conceptos ácido-base me ayudan a interpretar los fenómenos bioquímicos.

4. Cuando trabajo en grupo es más fácil aprender estos conceptos.

5. Valoro las discusiones porque me permiten poner a prueba las concepciones que poseo sobre estos conceptos.

6. La mejor forma de aprender los conceptos ácido-base es resolviendo problemas de lápiz y papel.

7. El aprendizaje de estas conceptos tiene relación con los fenómenos de la vida.

8. Una base es una sustancia capaz de donar un par de electrones.

9. Un ácido en solución acuosa produce iones $\mathrm{H}^{+}$

10. Lo mejor del aprendizaje de los conceptos ácido-base son sus prácticas de laboratorio.

11. Se me dificulta relacionar estos conceptos con la vida diaria.

12. Cuando realizo un experimento químico me preocupo por mi seguridad y la de mis compañeros.

13. Respeto las ideas y los puntas de vista de los demás así sean diferentes a las mías.

14. Los conceptos ácido-base difícilmente se pueden contrastar en el laboratorio.

15. Me gustan las prácticas de laboratorio cuando tengo que utilizar ácidos.

16. Una base es la sustancia que en solución acuosa puede formar iones hidroxilos $\mathrm{OH}$.

17. Un ácido es la sustancia que en solución dona electrones.

18. Estudio los conceptos ácido-base porque hacen parte del currículo.

19. Para poder explicar éstos conceptos, necesito relacionarlos con otras conceptos de la química.

20. El significado que tengo sobre los conceptos ácido-base es más válida e importante que el que tienen los demás.

21. Soy indiferente ante las dificultades de aprendizaje que tienen mis compañeros.

22. Entender las conceptos ácido-base me permite también comprender otros conceptos de la química.

23. Me es indiferente aprender los conceptos ácido-base. 
24. Una base es una sustancia neutra.

25. Una base es una sustancia que en solución adepta electrones.

26. Los conceptos ácido-base son difíciles de aprender.

27. Se me dificulta entender el significad de los conceptos ácido-base.

28. Participo con agrado en las discusiones cuando éstas me permiten llegar a acuerdos.

29. La determinación del pH de una solución requiere de instrumentos específicos.

30. Los conceptos ácido-base sólo se relacionan con la química.

31. Cuando un ácido reacciona con una base se produce un óxido.

32. Una solución es neutra cuando tiene un $\mathrm{pH}$ de 14.

33. Me gusta realizar experimentos sobre ácidos y bases únicamente por las cambios físicos que se observan.

34. Se me dificulta comunicar las dificultades que tengo para aprender conceptos nuevos como los de ácido-base.

35. El producto resultante de la reacción entre un ácido y una base se puede separar por métodos físicos.

36. Cuando mis compañeros no entienden un tema estoy dispuesto a colaborarles con mis explicaciones. 